\title{
Caminar desechos. Reflexiones desde las superficies de Aguabuena
}

Fouler les déchets. Réflexions à partir des surfaces d'Aguebuena

Walking waste. Some lessons from the surfaces of Aguabuena

\section{Daniela Castellanos}

\section{(2) OpenEdition}

\section{Journals}

\section{Edición electrónica}

URL: https://journals.openedition.org/bifea/11554

DOI: 10.4000/bifea.11554

ISSN: 2076-5827

\section{Editor}

Institut Français d'Études Andines

\section{Edición impresa}

Fecha de publicación: 1 abril 2020

Paginación: 41-62

ISSN: 0303-7495

\section{Referencia electrónica}

Daniela Castellanos, «Caminar desechos. Reflexiones desde las superficies de Aguabuena», Bulletin de I'Institut français d'études andines [En línea], 49 (1) | 2020, Publicado el 08 agosto 2020, consultado el 24 agosto 2021. URL: http://journals.openedition.org/bifea/11554 ; DOI: https://doi.org/10.4000/bifea. 11554

\section{(2) $(\mathcal{Q} \Theta \Theta$}

Les contenus du Bulletin de l'Institut français d'études andines sont mis à disposition selon les termes de la licence Creative Commons Attribution - Pas d'Utilisation Commerciale - Pas de Modification 4.0 International. 


\title{
Caminar desechos. Reflexiones desde las superficies de Aguabuena
}

\author{
Daniela Castellanos*
}

\begin{abstract}
Este artículo se centra en desechos que son fragmentos cerámicos y caminos que atraviesan la geografía y emergen o se desvanecen gracias a la acción conjunta de fuerzas humanas y no humanas, o a la falta de ella. Son detalles del mundo material que construyen y actualizan los alfareros de Aguabuena (Andes colombianos) con sus prácticas. Además de formas, pueden ser vistos como un concepto que nos invita a pensar nuestras categorías de conocimiento antropológico y arqueológico. En un recorrido que involucra mi experiencia investigativa de dos décadas entre este grupo humano, intento desarrollar una reflexión teórica sobre las superficies que son materia y método.
\end{abstract}

Palabras clave: alfareros, Aguabuena, desechos, superficie, Andes colombianos

\section{Fouler les déchets. Réflexions à partir des surfaces d'Aguebuena}

Cet article s'intéresse aux déchets, c'est-à-dire aux fragments de céramique et aux chemins qui traversent la géographie et émergent ou s'évanouissent grâce à l'action conjointe des forces humaines et non humaines, ou à son absence. Ce sont des détails du monde matériel que les potiers d'Aguabuena (Andes colombiennes) construisent et actualisent grâce à leurs pratiques. En plus des formes, elles peuvent être vues comme un concept qui nous invite à réfléchir sur nos catégories de connaissance anthropologique et archéologique. Dans un parcours qui implique mon expérience de recherche de deux décennies au sein de ce groupe humain, j'essaye de développer une réflexion théorique sur les surfaces comme matière et méthode.

Mots-clés : potiers, Aguabuena, desechos, surfaces, Andes colombiennes

\section{Walking waste. Some lessons from the surfaces of Aguabuena}

This article explores waste — ceramic fragments and the paths that they follow crossing mountains, emerging or disappearing through joint action of human and non-human forces (or its absence). They are parts of the material world constructed and acted upon by Aguabuena potters in the Colombian Andes. In addition to being thought of as forms, they can be seen as a concept that invites us to

* Departamento de Estudios Sociales. Universidad Icesi, Pance, Cali - Colombia. E-mail: Daniela Castellanos dcastellanos@icesi.edu.co 
reflect upon the construction of anthropological and archaeological knowledge. Journeying through my own research experiences among the potters for over two decades, I attempt to develop theoretical reflection concerning surfaces as matter and method.

Keywords: potters, Aguabuena, waste, surface, Colombian Andes

\section{INTRODUCCIÓN}

Este artículo conjuga, por un lado, aspectos de la experiencia de vida de los alfareros de Aguabuena (Andes colombianos) y de lo que han significado alrededor de dos décadas de investigación de campo de corte etnográfico con ellos ${ }^{1}$, y por otro lado, detalles del mundo físico que este grupo habita y ayuda a construir, esto es, de su materialidad. Específicamente, me centraré en desechos: fragmentos cerámicos y caminos que atraviesan la geografía y emergen o se desvanecen gracias a la acción conjunta de fuerzas humanas y no humanas, o a la falta de ella. Mi intención es pensar a través de huellas del mundo alfarero para reconsiderar nuestras formas de conocerlo. Me interesa retomar detalles del espacio cotidiano y de los elementos materiales con los que los ceramistas crean y actualizan lazos, con la intención de considerar cómo estas formas encierran en sí mismas otras formas-ideas que desafían nuestros métodos y nuestra aproximación a la alfarería y su registro. Para ello, me sitúo desde un contexto específico y reflexiono de forma retrospectiva sobre mi propia experiencia investigativa y las formas en que mis preguntas han ido cambiando.

Siguiendo desechos (algunos de los cuales son fragmentos cerámicos), planteo una serie de reflexiones sobre la discontinuidad que invitan a repensar los sitios y contextos arqueológicos más allá de unidades necesariamente cerradas y continuas, en la memoria que no es ordenada y progresiva sino fragmentada y saltuaria, y en esa medida en la revisión de las aproximaciones que suponen una relación directa a nivel histórico entre prácticas y sujetos situados en el presente etnográfico y aquellos del pasado colonial o prehispánico. También en los recorridos y tránsitos por superficies que se interrumpen. Así, en lo que sigue, apuesto por derivar desde los desechos, una lectura distanciada de una imagen coherente del oficio de alfarero y del taller cerámico y predominantemente cimentada sobre la suposición en la pervivencia de tradiciones de larga data y relaciones estáticas. Con esto no sugiero un panorama en que todos los eventos son coyunturales y evanecentes, sino que propongo considerar las acciones de los desechos en el mundo para permitirnos aprender de superficies que si bien son discontinuas y cambiantes, también tienen cierta profundidad temporal.

1 Inicié mi investigación en Aguabuena en 2001. Desde entonces he realizado varias temporadas de campo de distinta duración, combinando metodologías y técnicas arqueológicas y etnográficas que iré detallando a lo largo del texto. 
Mi propuesta se alinea con otros trabajos que, 'pensando a través de las cosas' ponen en el centro la materia para trazar puentes entre teoría y método desde una aproximación etnográfica que deja que las cosas hablen (cf. Henare et al., 2007). En este escenario, los objetos son más que vehículos o el telón de fondo de eso que llamamos cultura. En cambio, éstos importan porque tienen un estatus ontológico que es el punto de partida de nuestros cuestionamientos y marcan el camino para explorar la realidad, desplegando una estrategia analítica. Más que simples objetos o materia, son también conceptos (cf. Gordillo, 2014). Con esto en mente, los desechos nos hablan desde su materialidad. Se trata entonces de valorar sus formas para ser más que la base de clasificaciones tipólogicas, en el caso de los desechos que son fragmentos cerámicos, o rasgos del paisaje, cuando son desechos que son caminos. Reconocer sus efectos en el mundo desde sus quiebres y fracturas, o su potencialidad para desaparecer o emerger, nos da la posibilidad de vislumbrar otros asuntos relativos a las discontinuidades que conectan, como su duración y las cualidades de su presencia en el espacio.

¿Qué nos enseñan los alfareros desde sus prácticas sobre las formas en que nosotros los antropólogos conocemos? Y más allá, ¿a qué tipos de problemas epistemológicos nos podrían enfrentar los desechos considerados como conceptos? Propongo explorar estas preguntas en lo que sigue para aportar a la discusión que convoca este dossier, esto es, el uso de la analogía etnográfica en estudios sobre comunidades alfareras del pasado2. Concretamente, me referiré a dos problemas que se derivan de mi observación sobre las acciones (y afectaciones) que propician los desechos en ese mundo desde las (des)conexiones a través de caminos y redes que se actualizan, emergen o desaparecen: primero, la unidad de un espacio habitado-transitado (llámese sitio, taller, camino, comunidad) siempre en fragmentación y segundo, el problema del contexto en un mundo en donde muchas cosas están descontextualizadas.

\section{ALFAREROS Y TEORÍA SOCIAL CONTEMPORÁNEA}

Autores como Gosselain (1999) y Barley (1994) proponen que la producción cerámica es un sistema de pensamiento que expresa prácticas sociales, culturales y valores simbólicos que crean, reproducen y desafían el orden social. Así, el oficio de transformar la cerámica cruda en cocida es más que una actividad productiva. Representa un modelo de mundo (o un 'modelo de mundo alfarero', como lo llamó Barley) que inscribe relaciones de género, parentesco, política, ecología y cosmología; trasciende a aspectos relativos a la sexualidad, la identidad y las emociones, e incluso informa nuestra relación con la materia. En suma,

2 Algunas de estas ideas fueron presentadas en el taller internacional ¿Qué es una Relación? Perspectivas Etnográficas desde una Sudamérica Indígena en la Universidad Católica de Chile (Santiago, 2017), en el Encuentro de Teoría Arqueológica en Ibarra (Ecuador, 2018) y en el XVII Congreso de Antropología en Colombia realizado en la Universidad Icesi (Cali, 2019). Agradezco a todos los expositores de estos encuentros, de quienes recibí preguntas y comentarios que contribuyeron a refinar las reflexiones aquí planteadas. 
la propuesta de los autores mencionados permite pensar el valor que tiene la alfarería en sí misma como una experiencia de estar en el mundo que comparten ciertos grupos humanos a través del tiempo.

En Colombia, y más específicamente en los Andes orientales, los estudios sobre alfareros han enfatizado los aspectos estéticos y técnicos de sus prácticas y generalmente han quedado como apéndices de trabajos arqueológicos o etnoarqueológicos que sirven para demostrar la continuidad y correspondencia entre las técnicas precoloniales y actuales (Broadbent, 1974; Falchetti, 1975; Mora de Jaramillo, 1974; Therrien, 1991). Si bien otros estudios etnográficos han incluido temas distintos a los económicos (por ejemplo, las interrelaciones entre la organización social del trabajo productivo y las divisiones de género), estos trabajos han reforzado una visión esencialista y estática de los alfareros del presente al ponerlos como un espejo retrovisor para indagar sobre la producción del pasado colonial y precolonial (cf. Duncan, 1998), sin aparente derecho al cambio o la modernización (cf. Vasco, 1987).

Lo anterior puede contrastarse con un campo de estudio más nutrido en el que a veces se incluye a los alfareros: el campo de los artesanos. Al respecto, es más fácil encontrar trabajos preocupados por entender la incidencia de las prácticas de los artesanos en el mundo contemporáneo (Cant, 2019; Herzfeld, 2004; Kondo, 1990; Koptiuch, 1999). El trabajo de Herzfeld entre aprendices de zapatero en Creta es diciente en su intento por derivar reflexiones teóricas a partir de la situación actual de los artesanos. Su tesis de la «jerarquía del valor global» analiza la forma en que estos grupos se incorporan en y participan del mundo globalizado, mostrando su marginalidad como parte de una tensión entre lo moderno y lo tradicional, y su incidencia en la construcción de la imagen de estado-nación de la Grecia contemporánea (Herzfeld, 2004). En esa misma línea, investigaciones de Chaves \& Nova (2014) brindan una lectura de las artesanías étnicas en contextos colombianos disímiles como la Amazonía o la Guajira, analizando los procesos de patrimonialización de ciertos objetos, junto con la activación del turismo. Otros estudios más recientes, como el de Alana Cant entre talladores de madera de México (2019), exploran el campo estético como una intersección entre productores y consumidores de arte y los mutuos condicionamientos que estos procesos imponen sobre el mundo social de los artesanos. En particular, se interesa por cómo distintos actores que participan de «lo estético» definen su pertenencia a dicho campo a partir de la competencia, o el establecimiento de alianzas y comunidades políticas en disputa por el reconocimiento a la propiedad intelectual y los derechos de autor.

Otro tema a propósito de los artesanos ha sido el del aprendizaje de técnicas desde una lectura amplia de la tecnología como tekhne, o saber hacer (Ingold, 2000: 294), y el interés por los aspectos sociales relacionados con el desarrollo de las habilidades y las destrezas de quienes transforman la materia y, a su vez, son transformados por esta. Aquí, el aprendizaje ha servido como teoría y método de trabajo para captar, a través del propio cuerpo del investigador, aspectos del trabajo artesanal que no se comunican por medios convencionales y que están relacionados con el 
conocimiento implícito que se adquiere a través de la experiencia (Coy, ed., 1989). Así mismo, el aprendizaje se vuelve una ventana para captar las relaciones de poder en las que se inscriben las relaciones maestro y aprendiz, además de otros aspectos como las construcciones de género y subjetividades que se validan en estrecha relación con un oficio. Por ejemplo, el trabajo de Trevor Marchand entre constructores de adobe en África explora los modos en que el ejercicio de un oficio marca una interrelación vital de los sujetos con el mundo que los rodea, enlazando cuerpo, mente y entorno (Marchand, 2010).

Esa interrelación vital y reflexiva del artesano con el mundo ha sido explorada en la obra de Richard Sennet El artesano (2008) —el primero de una trilogía dedicada a la cultura material— desde su atención en el hacer. «¿QQué nos revela el proceso de hacer cosas concretas sobre nosotros mismos?» (Sennet, 2008: 8), se pregunta el autor como antesala a un recorrido histórico y filosófico interesado en las cosas que se hacen, los procesos que organizan ese hacer, la participación de los individuos y grupos en esto y sus implicaciones más sociológicas y políticas. El oficio del artesano tiene que ver con la habilidad de «hacer las cosas bien» (Sennet, 2008: 8), lo que involucra el desarrollo de una técnica vinculada a una forma particular de ser y estar en el mundo: es, por lo tanto, un conocimiento corporal y moral al tiempo. La obra de Sennett confiere al arreglo práctico del hacer una dimensión existencial que reivindica la materia al ponerla en el mismo plano que el pensamiento. Mi texto también reconoce este estatus que le da Sennett a la materia, pero esta vez no solo conferido a las cosas completas que han sido 'bien hechas', sino también a aquellas que, estando rotas, de alguna forma son abortadas o rechazadas $-y$, sin embargo, no dejan de existir-. Precisamente desde estos márgenes de lo abandonado, expulsado o simplemente no vuelto a cuidar, también podemos seguir interrogándonos por quiénes somos.

En lo que sigue, quiero proponer una reflexión de los alfareros y su mundo interesada en sus desechos. Más que pensar desde la alfarería como una actividad social y económica inscrita en redes globales o situarme en la subjetividad de los alfareros y sus formas diferenciadas de aprender el oficio, mi análisis se centrará en rasgos de la materialidad de Aguabuena que reflejan detalles que los ceramistas (des)hacen y con los que construyen, actualizan o van borrando su lugar en el mundo.

\section{ENCONTRANDO FRAGMENTOS QUE SON DESECHOS}

Miles de fragmentos cerámicos de todos los tamaños y distintos períodos de tiempo yacen en las superficies de Aguabuena, un sector rural en la falda de un cerro comprendido entre dos veredas 3 , Candelaria Occidente y Pueblo Viejo, en el municipio de Ráquira en la cordillera oriental de los Andes colombianos (fig. 1). También, estos fragmentos se encuentran en los caminos que conectan

3 Vereda es el término usado en Colombia para referirse a la subdivisión de un territorio rural en el mapa de organización política y administrativa de un municipio. 


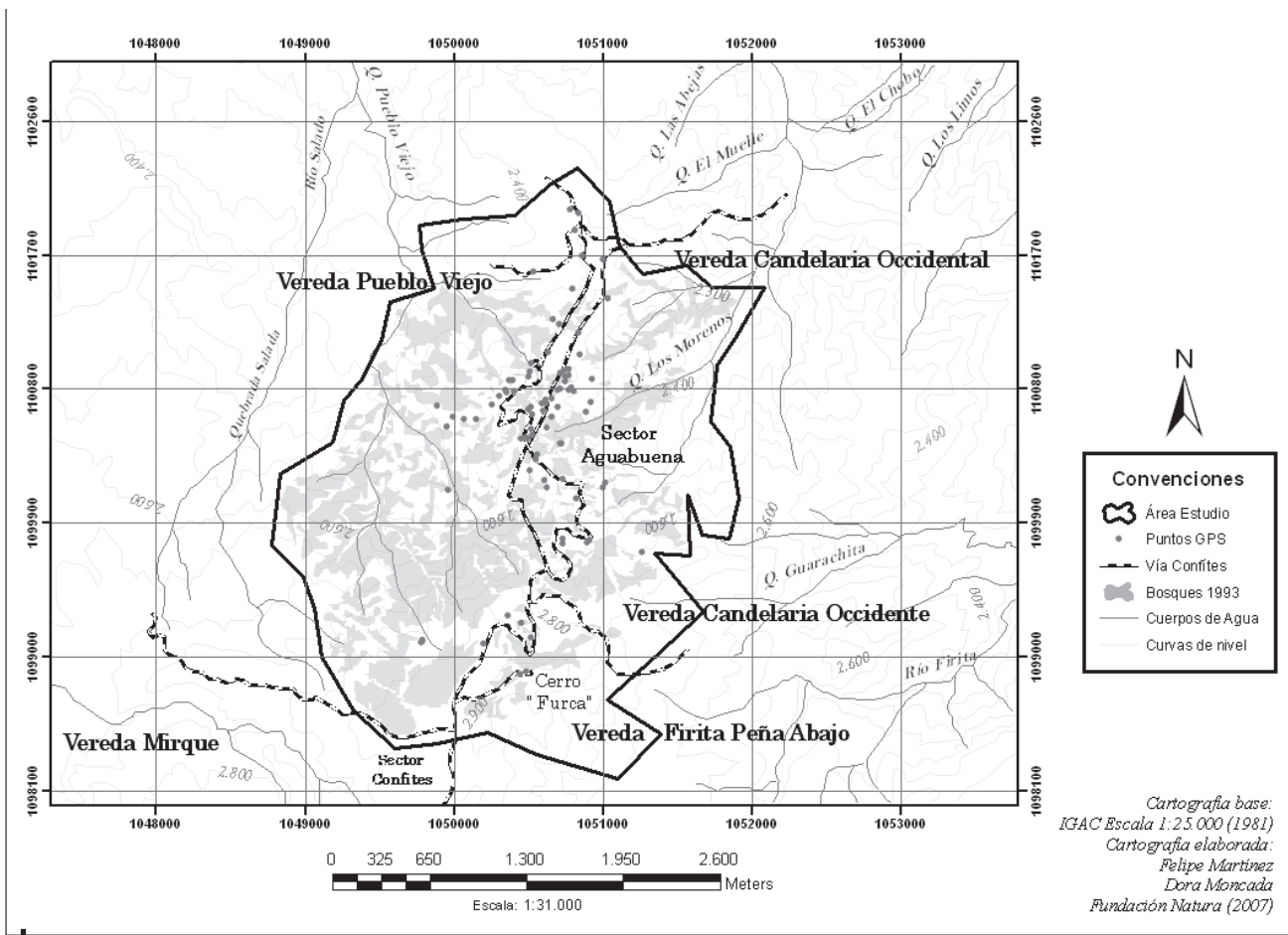

Figura 1 - Localización geográfica del sector de Aguabuena en Ráquira (Boyacá, Colombia)

Los puntos GPS corresponden a la localización de talleres cerámicos activos en el año 2006

(C) D. Moncada Rasmussen (2010: 277).

Aguabuena con el resto de veredas y puntos en la geografía 4 . Su presencia es tan ubicua que se vuelven un detalle que pasa a ser fácilmente desapercibido. Se les puede encontrar apilados en montones y puestos en lugares discretos dentro de los talleres cerámicos, o al lado de los caminos, algunas veces como parte de improvisadas paredes que rompen el viento, o para ser reciclados como materiales de construcción de casas campesinas de adobe. También sirven como relleno de los huecos del pavimento de carreteras secundarias por donde transitan carros, buses y motocicletas. Finalmente, están debajo de la tierra, como parte del suelo. Pese a las inclemencias del clima, o las actividades humanas y animales, los fragmentos persisten y permanecen, fragmentándose con el tiempo, esculpiendo la superficie, añadiéndole textura y color a un paisaje montañoso y erosionado. Allí, la basura cerámica permanece en la superficie, como puntos discretos en el espacio, y a veces como una superficie en sí misma, como cuando construye canales a través de los cuales se mueve y transita la vida (piénsese en los pies de los alfareros que recorren a diario los caminos cimentados por sus propios fragmentos cerámicos).

4 Estos caminos también pueden desconectar, como ocurre en las temporadas invernales, cuando a causa de las fuertes lluvias se vuelven instransitables. 
Mi primer proyecto en Aguabuena en 2001 (con una duración en campo de 4 meses) me acercó a la multiplicidad de lugares en donde podían encontrarse y las funciones que cumplían los fragmentos cerámicos. En ese entonces nos interesaba documentar cómo un taller cerámico se convertía en registro arqueológico (Castellanos, 2004). Para ello, realicé un mapeo de áreas de actividad de talleres de producción cerámica doméstica de la actualidad, inventariando ubicación, distribución y estado de los objetos cerámicos, enfatizando áreas de descarte. Combiné estos registros con observaciones sobre el ciclo de vida de las vasijas (desde manufactura hasta descarte) y datos de la organización y división del trabajo en las unidades de producción. En ese estudio, consideré una muestra de ocho talleres de producción de tipo doméstica.

Este interés cultivó mi atención hacia los objetos y formas en que eran descartados, siempre pensando en qué de lo que observaba en el presente sería rastreable en el futuro y cuáles serían sus registros o correlatos materiales. Mi caso empírico daba cuenta de una vida dinámica de los objetos —incluso después de ser descartados-, pues casi siempre eran reutilizados a pesar de estar rotos o reducidos a fragmentos.

Así, un basurero cerámico en un camino, más que un área de actividad especializada, circunscribía en un punto discreto, miles de fragmentos de distintos talleres, dispuestos en distintos tiempos, y que a su vez cimentarían el camino. Esto fue claro el día que acompañaba a Marina con su carretilla llena de tiestos. Cuando ella arrojaba un buen número fragmentos sobre otros que yacían en la superficie, yo le pedí que nos detuviéramos antes de llegar a su casa. Entonces empezamos una conversación para mí reveladora.

Mientras inspeccionaba los materiales que estaban en el conjunto, tomé un asa de una jarra, y le pregunté sobre esta. «Ésta oreja es de Teresa», afirmó. «Mire la curva de la oreja y las dos líneas sobre la curva: son de la una de ella». Pese a su explicación esforzada, puesto que no es común que los alfareros se refieran verbalmente a cómo hacen las vasijas, no pude disimular mi cara de extrañamiento. Interpretando mi gesto, continuó: «la oreja es gruesa y el hueco ancho porque Teresa tiene dedos grandes y gruesos». Buscó otro fragmento, esta vez de ella, y expuso las diferencias entre ambos pedazos, considerando la anatomía de sus dedos y uñas y las improntas de sus formas corporales en la materia. Las palabras de Marina desplegaron un nuevo universo ante mis ojos. Desde entonces empecé a considerar la importancia de los detalles y la 'vida posterior' (el afterlife sensu Gordillo, 2014: 20-21) de los fragmentos siempre en perpetua fragmentación y que hacían de ese conjunto, muchos palimpsestos 5 .

Dentro de las posibilidades de la «vida posterior» de la materia, quisiera considerar la condición de los desechos en Aguabuena y su complicidad con el movimiento

5 En su interés etnográfico y espacial por los escombros dispersos en una de las áreas de agroindustria más importantes del norte de Argentina en el Gran Chaco, Gordillo introduce el problema de la vida después de un objeto que, como los escombros, está más cercano a la destrucción que a la creación. Tomando de Walter Benjamin la idea de «una vida posterior» (mi traducción) para pensar 
o el tránsito de distintos actores en sus recorridos. Desechos, además de ser una forma genérica de referirse a los residuos (por ejemplo, fragmentos cerámicos), se refiere también a caminos que surcan superficies quebradas, siendo ellas mismas superficies que surgen o desaparecen, dependiendo del tránsito a través de ellas. Allí confluyen viento, lluvia, pies en movimiento, terreno, erosión, humedad, pezuñas de animales de pastoreo, organismos y llantas de motocicletas. Todos juntamente esculpen caminos que se convierten en formas directas de llegada de un punto a otro a través de montañas. Despliegan una creación conjunta de actores humanos y no humanos y su complicidad con la materia. A veces, también desconectan, cuando se desvanecen, a falta de tránsito (fig. 2).

Otros caminos, para cruzar montañas y sin pavimentar, dependen de desechos para continuar conectando. Es el caso de carreteras cuyos huecos se tapan con pavimento artesanal o tiestos, o de caminos sobre los que yacen millones de

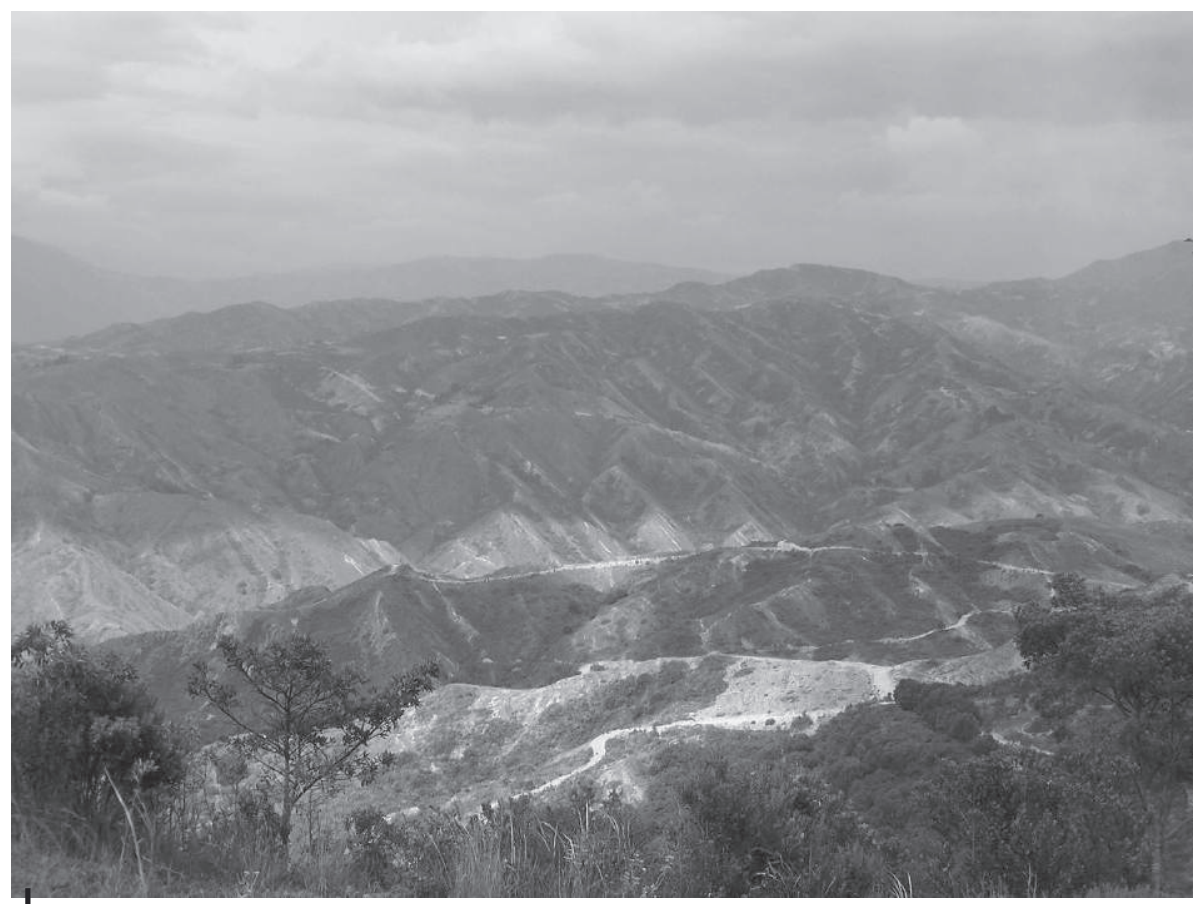

Figura 2 - Desechos que atraviesan la geografía quebrada

(C) Daniella Castellanos

que la vida no se limita a lo que tiene una «corporalidad orgánica» sino a «todo aquello que tiene una historia propia» (Benjamin, 1968: 71 en Gordillo, 2014: 20), el autor plantea la posibilidad que tiene algo inanimado, como los escombros, de tener también una «historia propia» (Gordillo, 2014:). Por ser un concepto que refiere una discusión filosófica más profunda, he decidido alertar al lector dejando el término en inglés y explicitando que debe entenderse desde el uso dado por el autor referenciado. 
fragmentos (fig. 3). Sobre esto último, cabe anotar que para recorrer las superficies de Aguabuena tuve que aprender a moverme a través de fragmentos cerámicos. Fue un reto pisar materiales desiguales, algunos con puntas filudas, otros de superficies curvas y resbalosas, que siempre craqueaban al recibir el impacto del peso de mi cuerpo sobre estos o tintineaban cuando por descuido los pateaba y los hacía colisionar con otros fragmentos que estaban cerca. De esto me percaté el día que acompañé a mi familia adoptiva a la procesión de Semana Santa, un recorrido de algo más de 6 horas que me ocasionó dolores en piernas y espalda al recorrer superficies desiguales (fig. 4). Los conductores de buses intermunicipales o motos también eran conscientes de las propiedades de estos caminos, y les evitaban en los meses de lluvia cuando se convertían en «pistas de jabón». Así, moverse involucraba sincronía entre materiales y cuerpos.

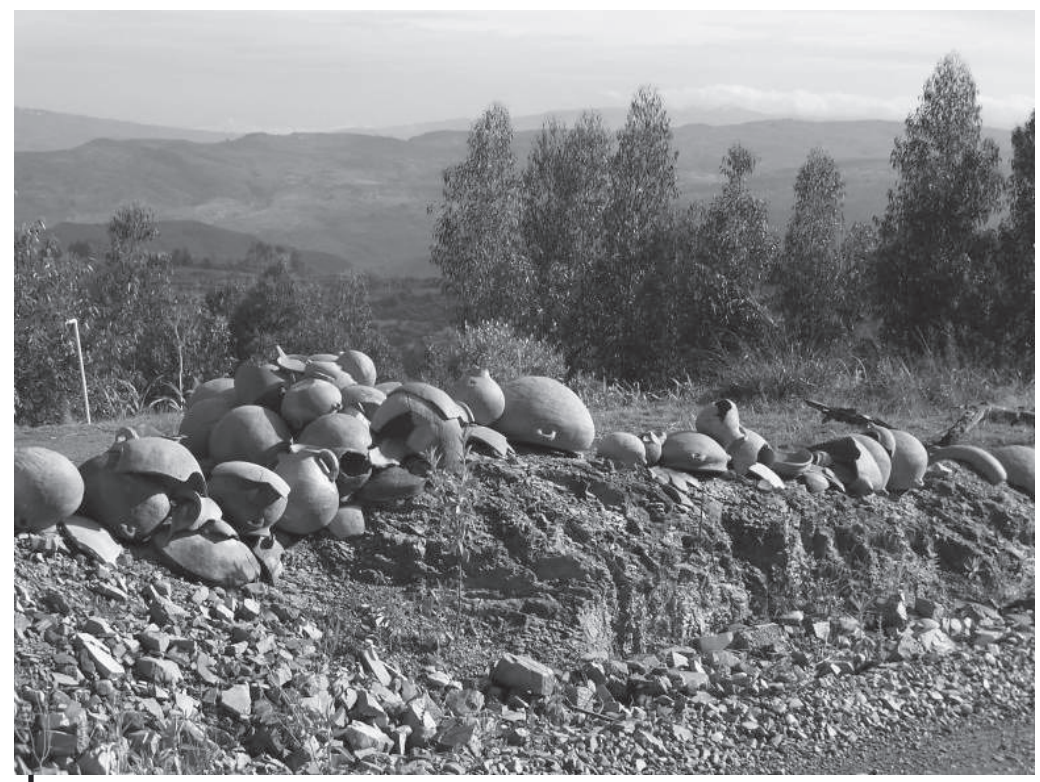

Figura 3 - Vasijas rotas y fragmentos cerámicos al lado del camino

(C) Daniella Castellanos

\section{AGUABUENA COMO PROBLEMA}

Aguabuena es, a la vez, un lugar físico y un grupo humano. Geográficamente se encuentra en la parte alta del municipio de Ráquira, sobre la falda de un cerro que está en un sector fronterizo entre dos veredas. En 2009, había alrededor de 120 personas agrupadas en unos 30 talleres cerámicos y distribuidas a lo largo de la montaña6. Desde muchos de los talleres se tiene una vista panorámica del

6 Actualmente, la población ha disminuido drásticamente por la muerte de los alfareros más viejos y la migración de los más jóvenes a los centros urbanos. En una corta visita a finales de 2019, se contabilizaron 12 talleres activos y una población de 25 alfareros. 


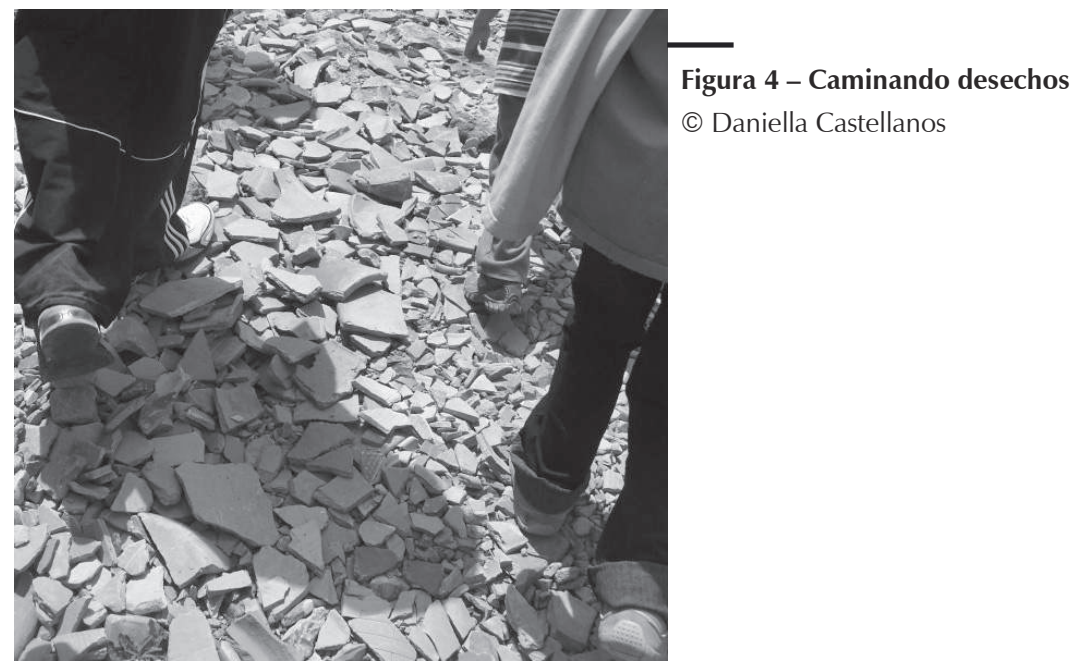

municipio, abajo en un valle, y se pueden observar las casas de los ceramistas de los alrededores.

Pese a que para la gente de la región, Aguabuena representa un claro referente espacial en la geografía del lugar, sus límites no son administrativos ni políticos (de hecho, no aparece en los mapas políticos de Ráquira), sino de tipo social (fig. 5). Originalmente, Aguabuena era un predio perteneciente a tres generaciones atrás de los actuales alfareros que se fue fragmentando a medida que la familia se fue ramificando. Los tatarabuelos heredaron a sus hijos y estos a los hijos de sus hijos y así, una porción de un territorio que terminó dividido en múltiples puntos representados en fincas de pocas hectáreas y cuya división aún no termina. Un espacio tan fragmentado, sin embargo, no se correspondía con un escenario de relaciones familiares divididas o lejanas. Al contrario, los lazos de parentesco siguieron actualizándose a través de alianzas matrimoniales entre primos y de lazos de compadrazgo. Esta cercanía incrementaba la competencia, al todos tener la misma actividad económica, la alfarería, y todos explotar un territorio con recursos limitados (escasez de agua, suelos poco fértiles para la agricultura). Aguabuena es entonces un grupo social considerado como el mismo tipo de gente: todos son alfareros, todos con la misma tecnología cerámica, todos parientes, todos ocupando un mismo espacio.

En una visita en 2006, Arturo Bautista, a mi pregunta por los límites de Aguabuena, respondió:

De largo va desde los Vergel (taller que está en la parte más arriba del cerro) hasta donde Justa Bautista, y de ancho alcanza a coger lo que agarra el filo de la carretera.

En general, todas las respuestas que recolecté en distintas temporadas de campo, siempre me refirieron un territorio dinámico, de límites variables. Aguabuena se contraía o expandía dependiendo de si sus alfareros migraban o retornaban, o de las cuentas que incluían casas abandonadas o recién construidas. 


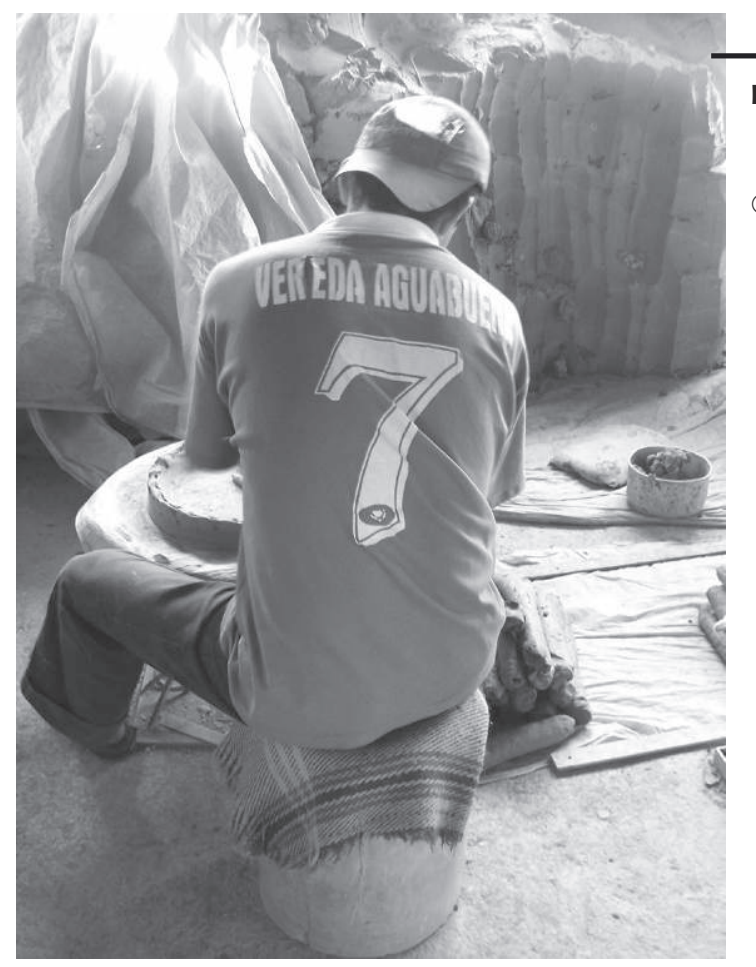

Figura 5 - «Vereda Aguabuena» en la camiseta del equipo de microfútbol de un joven alfarero

(c) Daniella Castellanos

Mi dificultad por fijar en un mapa una foto duradera de Aguabuena y sus alfareros, fue otro camino que me llevó a considerar las fragmentaciones, esta vez representada en cómo se (des)ocupa el espacio, cómo se mantiene (des)ocupado y por esta vía, lo artificioso de construcciones espacio-temporales totalizantes, cerradas y circunscritas. Además, a Aguabuena se llega, sale y recorre a través de desechos. Luego lo fragmentario, también literalmente marca los tránsitos de, hacia y dentro del lugar y las (des)conexiones de su gente.

A propósito de las ruinas de la provincia de Salta (Gran Chaco), Gordillo propone concebirlas como nodos que forman constelaciones (2014: 20). Retomando nuevamente a Benjamin y su concepto de constelación para pensar la Historia, la define como una «imagen de pensamiento» que evoca «una conectividad no casual definida por la multiplicidad, la ruptura, y la fragmentación» (2014: 20). Su carácter multicentrado, plástico, elusivo (pues no tiene fronteras claramente demarcadas), le permite considerar un mundo en el que los escombros, más que las ruinas, configuran una materialidad que aunque disruptiva (y en ese sentido con una connotación negativa), está cargada de afectos y es intrínseca a la vida de ese lugar (y de muchos otros).

Las ideas de Gordillo resuenan para el caso de Aguabuena y su naturaleza de fragmentos en fragmentación. ¿Cómo demarcar como una unidad, un sitio que está desecho? en otras palabras, lo que está roto o por romperse emerge con dinamismo y redefine los límites del grupo social entendido como comunidad, el 
territorio que habita y su materialidad característica7. Esta necesidad de construir bordes definidos para establecer lo que entendemos por «campo» en el trabajo etnográfico ya ha sido cuestionada; en cambio, se ha propuesto entenderlo como un constructo multiespacial y multitemporal, cuyas fronteras son sobretodo un asunto de conveniencia metodológica (Candea, 2007). A este ejercicio se enfrentan siempre los arqueólogos, al definir un sitio arqueológico que, de entrada, es un compendio de rasgos, objetos, huellas, materiales, cuya disposición nos recuerda la definición de constelación de Benjamin.

\section{4. (DES)CONTEXTUALIZAR}

En Ráquira, la alfarería fue el oficio principal de la población a la llegada de los españoles (cf. Orbell, 1995; Therrien, 1991; Falchetti, 1975; Mora de Jaramillo, 1974; Hernández Rodriguez, 1978; Broadbent, 1974) y se extendió hasta «los tiempos históricos recientes como ocupación de la capa rural baja al lado de la actividad agrícola» (Mora de Jaramillo, 1974: 20). Tal importancia contrasta con una falta de información histórica sobre el oficio a través de los siglos y la poca evidencia de su presencia a gran escala antes de la conquista. Por ejemplo, las crónicas y documentos oficiales de los visitadores, oidores y demás autoridades españolas, no brindan muchos detalles sobre los alfareros o el tipo de producción cerámica, y se limitan a hablarnos de «pueblos de olleros» en donde una población, (por cierto no numerosa y pobre), estaba «reducida a trabajar la loza» como forma de pagar el tributo que debían (Orbell, 1995: 20). Para compensar los pocos datos ethnohistóricos, algunos trabajos arqueológicos han aportado información sobre la posible antigüedad, escala y transformaciones en el tiempo de la producción cerámica de la zona.

El trabajo más importante fue realizado en los años 70 por Ana María Falchetti a través de una prospección intensiva y excavaciones arqueológicas que identificaron cuatro sitios arqueológicos con una baja densidad de material cerámico en Ráquira y sus alrededores (Falchetti, 1975). Los hallazgos contrastaban con la expectativa de encontrar una alta densidad de material arqueológico precolonial que permitiera documentar la antigüedad del oficio y sus desarrollos en la zona antes y después de la conquista y colonización españolas.

7 En The trouble with community (2002), Amit \& Rapport problematizan la «comunidad» como unidad analítica por excelencia de disciplinas como la antropología, mostrando cómo su centralidad ha oscurecido experiencias de grupos e individuos que rebazan los límites fijos y cerrados que la categoría impone. Para los autores, fenómenos como la globalización, la migración o los movimientos sociales, entre otros, nos enfrentan teórica y metodológicamente a la fragmentación espaciotemporal de este tipo de unidades cerradas. En ese mismo sentido, el «problema» de Aguabuena también nos enfrenta a los límites de nuestros conceptos para dar cuenta de las disrupciones, quiebres y transformaciones de nuestras unidades de análisis a partir de las dinámicas mismas de la gente en el territorio (por ejemplo, sus movimientos migratorios) y de la materialidad de este territorio. 
Por otra parte, tanto Falchetti como Sylvia Broadbent (1974) documentaron las técnicas de manufactura y quema que, por los años 70, seguían los alfareros de la zona rural de Ráquira. Ambas autoras establecieron continuidades y transformaciones en las técnicas y tecnología cerámicas descritas a partir de un tipo de cerámica arqueológica del siglo X y XI d. C., reportada por Falchetti en una de sus excavaciones en un municipio cercano a Ráquira (Falchetti, 1975: 199). Se trataba de un material de pasta dura con desgrasante de arena y una superficie con ranuras paralelas producidas al arrastrar partículas del desgrasante en el proceso de alisado de las vasijas, similar al tipo de cerámica «loza de arena» producida por los alfareros por ellas estudiados (Broadbent, 1974: 232). En los años 90, el trabajo de Therrien (1991) combinó la excavación de un basurero de un taller cerámico colonial y el trabajo con alfareros actuales, reforzando la tesis de la continuidad de las técnicas cerámicas entre el pasado colonial y el presente etnográfico del siglo XX.

Desde lo anterior, la producción cerámica de Ráquira aparece como continua y representativa a través del tiempo, así su registro arqueológico no lo sea. Distintos observadores en el tiempo (cronistas, viajeros, etnógrafos, arqueólogos, alfareros), han contribuido a construir esta continuidad, de la que en cambio no nos hablan los depósitos arqueológicos explorados.

En su estudio sobre las construcciones temporales de los arquéologos, Simonetti se refiere a una correlación entre la tendencia corporal de los arqueólogos de mirar hacia abajo, una comprensión vertical de la cronología y la conceptualización del tiempo como «profundidad». Así, los arqueólogos hacen presente lo que está ausente mientras interactúan con materiales en movimiento. Esta capacidad que él Ilama «sentir hacia adelante» es un ejercicio multisensorial que marca un movimiento hacia el futuro y hacia el pasado que está enterrado, una habilidad de proyectar los sentidos más allá de lo que es visible en la superficie (Simonetti, 2015: 70).

El criterio validador es la gravedad, bien sea a través de la estratigrafía como garante del paso del tiempo en los depósitos arqueológicos o a través de la mirada que de la superficie inspecciona hacia abajo cuando se enfrenta a esos depósitos o se mueve de abajo hacia arriba para construir cronologías. Esta condición también garantiza la presencia de un contexto crucial para interpretar el registro arqueológico y ha reforzado la idea moderna del pasado separado y desvinculado del presente, pues este está siempre escondido, enterrado y, por lo tanto, debe ser descubierto, para traerlo nuevamente a la luz (cf. Lucas, 2004; Harrison, 2011).

Es posible que una mirada guiada por lo anterior no considere o le reste relevancia a los materiales o prácticas en superficie porque escapan a la profundidad temporal. De hecho, uno de los problemas de los registros arqueólogicos superficiales es que pueden estar descontextualizados, pues pueden presentar alteraciones al no estar cerrados o claramente delimitados, como aparentemente sí lo están aquellos que se encuentran bajo tierra.

Más que volver sobre la dicotomía profundo/superficial, dos de los tropos fundamentales en el ejercicio arqueológico (González-Ruibal, 2011), me interesa 
reexaminar el caso de la producción cerámica de Ráquira partiendo de una consideración sobre la superficie y su profundidad, pero no desde una imagen genérica o ideal, sino desde una superficie de desechos como la que transité a diario con los alfareros de Aguabuena y que ellos ayudan a moldear. Esto me permitirá tender puentes entre antropología y arqueología a partir de mi propia experiencia investigativa.

\section{SUPERFICIES PROFUNDAS}

Tal vez como parte de mi entrenamiento como arqueóloga desarrollé una disposición corporal de caminar siempre mirando hacia abajo. Este hábito hizo que rápidamente me percatara de la cantidad de fragmentos cerámicos y vasijas rotas presentes en las superficies de Aguabuena, una redundancia que los convertía en elementos poco importantes. Esta irrelevancia era incrementada por su condición de materiales del presente, como pude notar ante la mirada poco interesada de mis colegas arqueólogos que me visitaron en el campo durante mi primera temporada de trabajo, a comienzos de los años 2000.

Por ese entonces emprendí un trabajo etnoarqueológico para refinar metodologías arqueológicas orientadas al estudio de contextos de producción cerámica, para contribuir al poco conocimiento de estos registros en el pasado precolonial y colonial. Como otros trabajos de los años 70, documenté las técnicas de manufactura y quema de los alfareros con los que conviví, en parte para intentar establecer criterios de correspondencia entre las técnicas descritas en las piezas arqueológicas de la zona y las técnicas que habían sido descritas en el presente etnográfico de investigaciones previas o de la mía propia (Castellanos, 2004). Esta condición, pensaba, sería la base para poder lanzar comparaciones más robustas entre ese pasado precolonial y colonial y el presente observado.

Pese a mis intenciones de establecer relaciones de continuidad, encontré sobre todo desconexiones y fracturas. La minoría de alfareros continuaba haciendo la «loza de suelo» descrita en trabajos anteriores, mientras que la gran mayoría hacía cerámica decorativa, algunas con formas tradicionales, pero también con otras más modernas. Más modernos también resultaron algunos de los procedimientos de manufactura, por ejemplo, el empleo de molinos de tracción animal para preparar la arcilla (mezclando barro, desgrasante y agua) en remplazo del proceso conocido como «pisado del barro», o el horno con combustible de carbón mineral a cambio del horno «tipo mediterráneo» de leña y de la quema a cielo abierto. Por otra parte, los alfareros actuales no establecían vínculos con el pasado precolonial, no pensaban tener ancestros indígenas alfareros, y para algunos era más aceptable en cambio, que su tradición fuera de los monjes europeos Agustinos Recoletos que construyeron el monasterio de Nuestra Señora de la Candelaria a finales de 1500, en el valle del río Candelaria donde se levanta el cerro que los alfareros ocupan (Castellanos, 2004; 2007). En suma, la continuidad que otros autores establecieron entre el pasado y el presente era difícil de sostener a partir de mis propios datos. 
Además, la gran cantidad de desechos cerámicos, todos en superficie, dispersos o, a veces, concentrados densamente, pero casi nunca dispuestos en depósitos bajo tierra, reforzaban la situación de descontextualización arqueológica. Este era el caso de las huellas de unos «antiguos» que los alfareros nombraban a veces para referirse a los precursores de la cerámica que actualmente hacían. Las ruinas de talleres u hornos de leña, no distantes de los talleres actuales, eran frecuentemente asociados a estos «antiguos», así como la cerámica delgada, con granos gruesos de arena como desgrasante y hollín en la pasta por su uso para cocinar y que, frecuentemente, estaba asociada a estas estructuras. Los alfareros podían a veces nombrar a los antiguos refiriéndose a su relación de parentesco con ellos, o a veces ignorar su historia. «Antiguos» era un término ambiguo, pero a la vez era el referente más claro que había para nombrar a unos ancestros de un pasado reciente cuyas huellas, todas en superficie, y siempre presentes en sus trayectos cotidianos, parecían no interpelarlos ${ }^{8}$.

Esta situación contrastaba con los discursos oficiales promovidos por el gobierno municipal y nacional (a través del Ministerio de Cultura y Artesanías de Colombia), y reforzados por la imagen que los académicos contribuían a construir de un grupo de artesanos cuya producción cerámica era una tradición transmitida de generación en generación, como legado de un pasado prehispánico y un patrimonio cultural de todos los colombianos (cf. Ministerio de Cultura, 2014).

Ya otros autores se han referido a la forma en que construimos a nuestros grupos de estudio como unos 'otros' suspendidos en el tiempo, negándoles la posibilidad de la contemporaneidad con respecto a un 'nosotros' quienes observamos (Fabian, 1983). Aquí es también bienvenida una reflexión más reciente de Gosselain (2016: 218-219) sobre la etnoarqueología y su mirada cómplice con el evolucionismo, al instrumentalizar los grupos humanos del presente volviéndolos fósiles del pasado. Lo que observaba por ese entonces y de manera más acelerada cada vez que revisitaba Aguabuena, era un grupo de familias adaptando sus modos de producción, de manera rudimentaria, a una modernidad con la que se conectaban fragmentariamente a través de desechos.

Esa discontinuidad vista en la materialidad de Aguabuena, y también la memoria histórica de sus alfareros, me hizo pensar que la contribución de ese primer trabajo debería estar dirigida hacia la construcción de una analogía no del tipo histórica directa, sino «general comparativa» (Gould \& Watson, 1982: 357) e «institucional» (Sillar \& Ramón Joffré, 2016: 656), en tanto brindara un marco rico en detalles para interpretar aspectos generales de formas de organización social y económica del pasado, precisamente a través del contraste con las diferencias que el presente podría representar con respecto al pasado precolonial y colonial (Castellanos, 2004: 85-86).

8 Otros casos en los Andes, como el reportado por Lema \& Pazzarelli (2018), refiere los antigales, como casas o puestos abandonados y de interés arqueológico cuya importancia no era así reconocida por la comunidad Huachichocana (Argentina), y quienes con dicho término hacían una «manifestación genérica de "ancestralidad" hacia unos referentes materiales en el paisaje, que sin embargo, desaparecían en sus relatos» (2018: 114). 
A partir de la preocupación inicial de lo que es visible y cómo se fija en el registro arqueológico de un taller cerámico, me adentré en la pregunta por las huellas arqueológicas, tema que exploré en una segunda investigación en 2006. En ese entonces y a través de un trabajo de campo etnográfico breve de un mes, centré mi atención en la vida social de los alfareros y sus objetos, buscando identificar referentes materiales (huellas) de sus comportamientos y prácticas cotidianas y considerando su posible visibilidad arqueológica. Abordé aspectos rituales de la quema cerámica, de construcciones de género y sexualidad de los alfareros y cómo se manifiestan en las vasijas que hacen, y de experiencias de la vida cotidiana como la envidia. Combiné observación participante, levantamiento de historias de vida de alfareros (una muestra de 5 ceramistas, 2 hombres y 3 mujeres de distintas edades) e historias de vida de algunos objetos (por ejemplo, vasijas u hornos que, pese a estar rotas, se encontraban curadas, cumpliendo funciones distintas a su función original), diagramas de parentesco, cartografía social de Aguabuena, y un inventario fotográfico de vasijas producidas en talleres donde se levantaron las historias de vida.

Un trabajo posterior sobre la envidia de los alfareros me puso como problema el contexto, en tanto herramienta heurística para una teoría etnográfica sobre esta experiencia (Castellanos, 2015). El ejercicio fue resultado de una temporada de campo de un año (2009-2010), en el que exploré etnográficamente las representaciones y experiencias de la envidia entre los alfareros, los agentes que la experimentan y activan y sus manifestaciones materiales.

En Aguabuena, la envidia circulaba a través de redes de parentesco y de objetos que eran a su vez una red. Por ejemplo, mangueras de caucho que transportaban agua desde las fuentes hídricas hasta las casas a través de un sistema de acueducto artesanal. Siempre en movimiento, las causas de la envidia no eran importantes, sino sus manifestaciones y síntomas (Castellanos, 2015). Estos criterios de relevancia hicieron que indagara por la forma en que mis interlocutores construían contexto, lo que Dilley (2002: 439) ha llamado «la vida social de los contextos».

Mi propio aprendizaje de la alfarería, me permitió profundizar en la relación que construyen los alfareros con las vasijas (sesiones que registré con una cámara de video para complementar mis tradicionales notas de campo). Asumir el rol de aprendiz, me dio una perspectiva fresca y novedosa, desde mi propio cuerpo como instrumento de investigación, sobre aspectos verbalizados y no del oficio cerámico, el rol del género, el paisaje sonoro de un taller, además de permitirme renegociar mi estatus como investigadora en campo, esta vez dispuesta a dejar su acostumbrado lugar de observadora con lápiz y libreta, para «embarrarse» partes del cuerpo y su atuendo, tal como la mayoría de los demás.

A través de la envidia y sus canales de circulación, pude considerar conexiones y desconexiones que hacían los alfareros en los referentes con los que daban cuenta de su mundo. Su interés en indicios (cf. Ginzburg, 1989)9, encarnados en

9 Carlo Ginzburg rastrea el origen de lo que él llama el «paradigma indiciario», un modelo epistemológico central en las ciencias humanas de finales del s. XIX, a través del saber cinegético 
detalles de la materia (correlatos materiales, como diría un arqueólogo), me hizo considerar más seriamente la materialidad como materia prima (en sentido literal) de mis propias reflexiones teóricas. Así, la superficie tomó relevancia en tanto su textura y topología alteraban también el ritmo y dirección de la envidia. Esta atención desplegó otros detalles, antes redundantes, pero ahora vueltos relevantes. Considerar la importancia de las superficies tampoco es novedoso ni original. En arqueología, por ejemplo, siempre han sido importantes como parte de metodologías de terreno que complementan las excavaciones (Harrison, 2011)10. Más allá de este hecho, podemos pensar como nos dice Ingold, en que:

«La superficie puede ser observada en diferentes escalas, desde cerca y desde lejos, y cada una revelará diferentes texturas, granos y diseños (...) [apareciendo] como igualmente arrugada, moteada y poliforma (Ingold, 2018: 71).

Lo anterior es solo el llamado a tomarnos en serio la experiencia de estar sobre el suelo (cf. Ingold, 2018), lo que puede marcar para unos una mirada necesariamente orientada en (re)ensamblar continuamente sus múltiples elementos constitutivos como ejercicio metodológico (Harrison, 2011: 154-155).

Lo anterior y mi experiencia kinestética (Ingold, 2018: 71) recorriendo caminos de desechos, han inspirado la necesidad de dar cuenta de un mundo en el que los fragmentos son materia y también método. Son materia, porque existen objetivamente en el mundo y lo ayudan a construir, y son método, porque son el cómo transitamos ese mundo (fig. 6) ${ }^{11}$.

\section{FINAL, MÁS DES-HECHOS}

Los desechos son generalmente basura. Elementos que han sido descartados o abandonados y que tienen una valencia negativa (por ejemplo, los escombros a los que se refiere Gordillo, [2014]), en tanto materia cuya vida no es considerada

de los primeros cazadores que «tiene la capacidad de remontarse desde datos experimentales aparentemente secundarios a una realidad compleja, no experimentada en forma directa» (Ginzburg, 1989: 144). Este origen lo extiende más allá, vinculando diferentes ramas del conocimiento. Por ejemplo, emparenta el psicoanálisis de Freud, la literatura de Conan Doyle y la historia del arte desde el método morelliano con la medicina, gracias a que lo marginal e incluso invisible adquiere un aspecto revelador. En esta forma de conocimiento, los indicios son huellas o síntomas de algo más grande y potente.

El paradigma que reconstruye Ginzburg está difundido «por todo el mundo, sin límites geográficos, históricos, étnicos, sexuales o de clase» (1989: 164), y en su universalidad se incluyen formas de conocimiento como las de los alfareros de Aguabuena.

10 Otras superficies a las que les prestan especial cuidado los arqueólogos son las de los artefactos y objetos que analizan. Análisis traceológicos en artefactos líticos, o granulométricos en la cerámica, son solo algunos ejemplos de cómo las superficies, esta vez a una escala más detallada, encierran claves sobre lo que no está presente.

11 Reconocer que la materia, más que la composición objetiva del mundo, también es el cómo nos movemos en el mundo, no representa un esfuerzo intelectual mayor si nos situamos desde Aguabuena. Como he mostrado en el texto, esta conjunción entre materia y método literalmente ocurre en la doble valencia de los desechos. Más allá de que esto resulte (o no) evidente desde el 


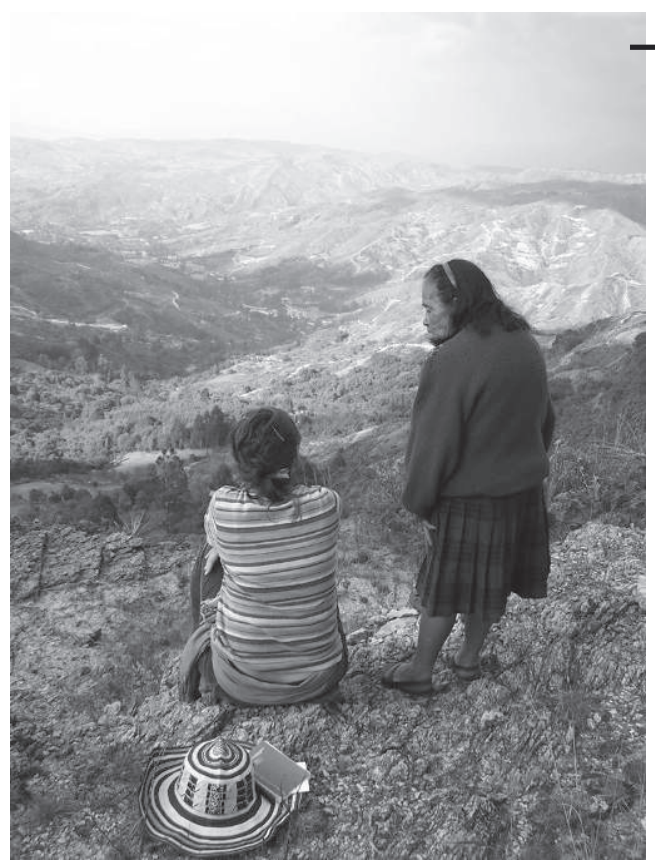

Figura 6 - Encontrando desechos

(C) Jan Grill

necesariamente útil y menos con una vida después de que han sido descartados. Sin embargo, los arqueólogos sabemos que lo que se descarta siempre es útil, tiene múltiples fines y una historia de vida que continúa.

Lo desecho contiene en su forma la acción del des-hacer, un movimiento progresivo pero no hacia adelante, sino hacia atrás de un presente que marca el momento cuando ese elemento es creado y existe como unidad objetiva. Cuando pienso en caminar a través de desechos, esta reflexión me invita a des-hacer mis pasos en una acción que trasciende horizontes temporales y no acaba. Eso posibilita que mucho de ese des-hacer lo haya hecho retrospectivamente (por ejemplo, desde el escritorio, mientras escribo estas líneas), objetivando mi participación en el campo, como paso necesario para una reflexividad que es condición de la objetividad científica (cf. Bourdieu, 2003). En ese recorrido hacia atrás, también encontré vacíos, asuntos inacabados, quiebres que hacen de esa posible acción del des-hacer un movimiento que, si bien idealmente es pensado como continuo y prolongado, en la práctica también incluye ausencias y discontinuidades.

¿Cómo vincular el pasado con el presente?, es una pregunta irresuelta y que nos desafía a muchos, en especial a aquellos que hacemos una etnografía interesada en lo que queda $y / o$ se desvanece, y por tanto en las improntas de la vida que transcurre en movimiento continuo, pero se fija en el tiempo a través de algo que llamamos registro arqueológico. Yendo más allá de las discusiones y críticas

punto de vista empírico, su consideración es un ejercicio por hacer de ese estatuto ontológico de las cosas, el verdadero punto de partida para interrogar la realidad que se nos presenta. 
a las formas como el presente se aliena en arqueología (Harrison, 2011: 149), creo entender que algunas respuestas podrían estar sugeridas en la materia misma que investigamos. Por ejemplo, si lo hacemos desde desechos, podríamos considerar relaciones discontinuas y en fragmentación como materia prima de representaciones menos estáticas y totales sobre los fenómenos que queremos entender (por ejemplo, la alfarería). El tener presente que los quiebres que vemos en el presente, más que formas pueden llevarnos a considerar las relaciones entre dos escenarios discontinuos (como lo son -incluso para los propios alfareros desde su misma historia social - el pasado prehispánico, colonial y el presente etnográfico de Aguabuena), nos permite incluir de manera importante las ausencias y fragmentaciones. Y si imaginamos que el camino vinculante entre pasado y presente fuera precisamente desechos, entonces nuestros compromisos intelectuales también estarían obligados a considerar las desconexiones o conexiones cuya duración es variable.

El coleccionismo de objetos y antigüedades ha acompañado desde siempre a la arqueología como práctica, esto es, antes de que fuera una disciplina propiamente institucionalizada (cf. Schnapp, 1996). Este protagonismo que desde siempre le hemos conferido a las cosas y sus materiales es el que he querido revivir en estas páginas. Sin embargo, mis aprendizajes con los alfareros estarían incompletos sin incluir las experiencias que me llevaron a interesarme en su mundo y cómo, en el proceso de irlo descubriendo, mis preguntas de investigación y formas de explorarlo fueron cambiando. Después de todo, llegué a Aguabuena recorriendo caminos mientras miraba hacia abajo, una disposición que me llevó a encontrar los desechos, gracias también a un ojo entrenado para verlos. Pero luego, en el campo, aprendí a transitar a través de ellos, comprendiendo cómo otros (los alfareros de Aguabuena) los transitan.

Caminar desechos y a través de desechos, no solo hizo que mi cuerpo se implicara en movimientos que iban apreciando el flujo incesante de la materia y su desenvolvimiento (cf. Ingold, 2012), sino que también fuera transformándose en estos recorridos. Si como he mostrado, los desechos son materia que también es método, entonces el mundo de los alfareros de Aguabuena se nos despliega como un escenario que, entre otras cosas, permite enriquecer nuestras propias apuestas epistemológicas. Esta reflexión no habría sido posible sin detenerse en cómo intervienen los desechos en vidas concretas. Hay razones de sobra en este texto para constatar que los desechos no son materia pasiva o por fuera de una relación; al contrario, siguen vigentes y actualizándose pese a ir fragmentándose para renovarse o desaparecer.

Finalmente, es usual encontrar reflexiones sobre el papel de la etnografía en arqueología. Este artículo es tal vez el testimonio de un recorrido a la inversa. Así, el camino dibujado en estas líneas muestra cómo mi trabajo etnográfico ha estado informado por una sensibilidad arqueológica, que me ha llevado, desde los inicios, a cultivar un gusto por los detalles de las formas materiales, considerando además su permanencia en el tiempo o su desaparición. Esta disposición, sin embargo, fue potenciada por mi encuentro con los alfareros de Aguabuena, quienes me 
han mostrado, a lo largo de este tiempo, otras posibilidades presentes en la materialidad. En ese sentido, el texto es un reconocimiento a cómo su mundo ha impactado el mío, al haberme permitido encontrar recursos que, además de empíricos, son analíticos. Si bien los desechos existen en muchos contextos, en Aguabuena tienen formas particulares. Pero su especificidad, más que un rasgo de exotismo, es suceptible de convertirse en dispositivo para pensar a través de, por ejemplo, lo más inmediato, como los caminos mismos de Aguabuena, o lo más trascendente, como las cualidades de una memoria y un tiempo discontinuos y su relación con las superficies en donde se manifiestan. En ambos casos, los desechos han sido el método.

\section{Referencias citadas}

AMIT, V. \& RAPPORT, N., 2002 - The Trouble with Community. Anthropological Reflections on Movement, Identity and Collectivity, 177 pp.; Londres Stirling: Pluto Press

BARLEY, N., 1994 - Smashing Pots. Works of Clay from Africa, 168 pp.; Londres: Smithsonian Instution Press.

BENJAMIN, W., 1968 - Illuminations: Essays and Reflections (H. Arendt, ed.), 288 pp.; Nueva York: Schocken.

BROADBENT, S. M., 1974 - Tradiciones Cerámicas de las Altiplanicies de Cundinamarca y Boyacá. Revista Colombiana de Antropología, 16: 223-248.

BOURDIEU, P., 2003 - Participant Objectivation. Journal of the Royal Anthropological Institute, 9 (2) (February): 281-294.

CANDEA, M., 2007 - Arbitrary Locations: In Defence of the Bounded Field-Site. Journal of the Royal Anthropological Institute, 13 (1): 167-184.

CANT, A., 2019 - The Value of Aesthetics. Oaxacan Woodcarvers in Global Economies of Culture, 208 pp.; Texas: University of Texas Press.

CASTELLANOS MONTES, D., 2004 - Cultura material y organización espacial de la producción cerámica en Ráquira. Un modelo etnoarqueológico, 95 pp.; Bogotá: Fundación de Investigaciones Arqueológicas Nacionales (FIAN), Banco de la República.

CASTELLANOS MONTES, D., 2007 - Huellas de la gente del cerro. Detalles etnográficos sobre estilo, ritos de paso y envidia en la formación de un contexto arqueológico. Tesis de Maestría en Antropología Línea de Arqueología (sin publicar); Bogotá: Universidad de los Andes, Departamento de Antropología.

CASTELLANOS, D., 2015 - The Ordinary Envy of Aguabuena People: Revisiting Universalistic Ideas from Local Entanglements. Anthropology and Humanism, 40 (1): 20-34.

CHAVES, M. \& NOVA, G., 2014 - Políticas patrimoniales, productores artesanales y economías de futuro. In: El valor del patrimonio: mercado, políticas culturales y agenciamientos sociales (M. Chaves, M. Montenegro \& M. Zambrano, compiladores): 67-100; Bogotá: Instituto Colombiano de Antropología e Historia.

COY, M. W. (ed.), 1989 - Apprenticeship: from theory to method and back again, 310 pp.; Albany: State University of New York Press.

DILLEY, R. M., 2002 - The Problem of Context in Sociocultural Anthropology. Language and Communication 22 (4): 437-456. 
DUNCAN, R., 1998 - The Ceramics of Ráquira, Colombia. Gender, Work and Economic Change, 233 pp.; Florida: Florida University Press.

FABIAN, J., 1983 - Time and the Other. How Anthropology makes its object, 205 pp.; Nueva York: Columbia University Press.

FALCHETTI, A. M., 1975 - Arqueología de Sutamarchán Boyacá, 268 pp.; Bogotá: Banco Popular.

GINZBURG, C., 1989 - Indicios. Raíces de un paradigma de inferencias indiciales. In: Mitos, emblemas, Indicios. Morfología e Historia, 138-175; Barcelona: Gedisa.

GONZALEZ-RUIBAL, A., 2011 - In praise of depth. Archaeological Dialogues. 18 (2): 164-168.

GORDILLO, G. R., 2014 - Rubble. The Afterlife of Destruction, xiii + 315 pp.; Durham y Londres: Duke University Press.

GOSSELAIN, O. P., 1999 - In Pots we Trust. The Processing of Clay and Symbols in SubSaharan Africa. Journal of Material Culture, 4 (2): 205-230.

GOSSELAIN, O. P., 2016 - To hell with ethnoarchaeology! Archaeological Dialogues, 23 (2): 215-228.

GOULD, R. \& WATSON, P. J., 1982 - A dialogue of the meaning and use of analogy in ethnoarchaeological reasoning. Journal of Anthropological Archaeology, 1 (4): 355-381.

HARRISON, R., 2011 - Surface Assemblages. Towards an Archaeology in and of the present. Archaeological Dialogues 18 (2): 141-161.

HENARE, A., HOLBRAAD, M. \& WASTELL, S., 2007 - Thinking Through Things: Theorising Artefacts Ethnographically, 248 pp.; Londres: Routledge.

HERNÁNDEZ RODRÍGUEZ, R. G., 1978 - De los Chibchas a la Colonia y a la República (del clan a la encomienda y al latifundio en Colombia), 326 pp. Bogotá: Ediciones Internacionales.

HERZFELD, M., 2004 - The Body Impolitic: Artisans and Artifice in the Global Hierarchy of Value, 269 pp.; Chicago y Londres: The University Chicago Press.

INGOLD, T., 2000 - The Perception of the Environment: Essays on Livelihood, Dwelling and Skill, 465 pp.; Londres: Routledge.

INGOLD, T., 2012 - Toward an Ecology of Materials. Annual Review of Anthropology 41: 427-442.

INGOLD, T., 2018 - La vida de las líneas, 233 pp.; Santiago de Chile: Ediciones Universidad Alberto Hurtado.

KONDO, D., 1990 - Crafting Selves: power, gender, and discourses of identity in a Japanese workshop, 354 pp.; Chicago: University of Chicago Press.

KOPTIUCH, K., 1999 - A Poetics of Political Economy in Egypt, 184 pp.; Minneapolis: University of Minnesota Press.

LEMA, V. S. \& PAZZARELLI, F., 2018 - Las formas de la historia: equívocos, relaciones y memorias en los cerros jujeños. RAU Revista de Antropología de la UFSCAR 10 (2): 105-125.

LUCAS, G., 2004 - Modern Disturbances: On the Ambiguities of Archaeology. Modernism/ Modernity 11 (1): 109-120.

MARCHAND, T. H. J., 2010 - Making knowledge: explorations of the indissoluble relation between minds, bodies, and environment. Journal of the Royal Anthropological Institute (N.S.): S1-S21.

MINISTERIO DE CULTURA, 2014 - Ráquira. De la casa a la olla, 44 pp.; Bogotá: División de Patrimonio, Ministerio de Cultura. Colección Los Cuadernos del Barro. 
MORA DE JARAMILLO, Y., 1974 - Cerámica y ceramistas de Ráquira, 90 pp.; Bogotá: Museo Arqueológico casa del Marqués de San Jorge, Banco Popular.

ORBELL, J., 1995 - Los herederos del Cacique Suaya. Historia colonial de Ráquira (15391810), 312 pp.; Bogotá: Banco de la República.

MONCADA RASMUSSEN, D. M., 2010 - Análisis espacio-temporal del cambio en los bosques de robre (Quercus Humboldtii Bonpl.) y su relación con la alfarería en Aguabuena. Revista Colombia Forestal 13 (2): 275-298.

SENNETT, R., 2008 - The Craftsman, 326 pp. New Haven y Londres: Yale University Press.

SILLAR, B. \& RAMÓN JOFFRÉ, G., 2016 - Using the present to interpret the past: the role of ethnographic studies in Andean archaeology. World Archaeology, 48 (5): 656673.

SIMONETTI, C., 2015 - Feeling Forward into the Past: Depths and Surfaces in Archaeology. Time and Mind. The journal of Archaeology, Consciousness and Culture, 8 (1): 6989.

SCHNAPP, A., 1996 - The Discovery of the Past. The Origins of Archaeology, 384 pp.; España: British Museum Press.

THERRIEN JOHANNESSON, M., 1991 - Basura arqueológica y tecnología cerámica. Estudio de un basurero de taller cerámico en el resguardo colonial de Ráquira, Boyacá; Bogotá: Universidad de los Andes, Departamento de Antropología. Tesis de Pregrado, sin publicar.

VASCO URIBE, L. G., 1987 - Semejantes a los dioses: cerámica y cestería Emberá-Chamí, 209 pp. Bogotá: Universidad Nacional de Colombia. 